\title{
Low-cost antimicrobial fortification of ultrasound coupling gel: An ergonomic innovation to combat sonology-acquired nosocomial infections

\author{
Philip Okere $^{1}$, Nneka Iloanusi ${ }^{1}$, Ukamaka Itanyi ${ }^{2}$, Moses \\ Ezea $^{3}$
} \\ Date Received: 17-Sep-2017 \\ Revision Received: 29-Jun-2018 \\ Date Accepted: 14-Jul-2018 \\ Correspondence: \\ nneka.iloanusi@unn.edu.ng \\ https://dx.doi.org/10.4314/mmj.v3111.8
}

1. Department of Radiation Medicine, Faculty of Medical Sciences, College of Medicine, University of Nigeria, Ituku-Ozalla Campus, Enugu, Nigeria

2. Department of Radiology, University of Abuja Teaching Hospital, Gwagwalada, FCT, Nigeria

3. Medical Microbiology Department, National Orthopaedic Hospital, Enugu, Nigeria

\begin{abstract}
Background

Recent outbreaks of highly contagious diseases have prompted hospital departments to adopt preventive hygiene protocols. Use of shared equipment, including ultrasound transducers and coupling gels, potentially exposes patients to these microbes. Inexpensive means of microbicide fortification of plain/non-sterile ultrasound gel may be useful in interrupting nosocomial infections. The purpose of this study was to evaluate the effectiveness of low-cost antimicrobial fortification of ultrasound coupling gel in preventing nosocomial infections during ultrasound examinations.

Methods

Volunteer patients, 20 in number, who presented for an ultrasound scan in a busy radiology clinic in Enugu, Nigeria, were randomly divided into 2 groups of 10 each and were scanned using plain non-sterile gel and gel-fortified with $0.5 \%$ chlorhexidine and $70 \%$ ethyl alcohol (in a volume ratio of 20:2:1) respectively. Swabs were taken from the patients' skin, gel-laden transducer, and the cleaned transducer and subjected to microbiology analysis. Subsequently, plain and fortified gel samples were allowed to stand in their respective dispensers for 72 hours. The plain and fortified gel samples were subjected to microbiology analysis. Fisher's Exact Test was utilised to compare outcomes in the 2 groups of volunteers.

Results

With fortified gel, swab cultures from patients' skin and gel-laden transducer, and from the cleaned transducer, significantly yielded no growth $(\mathrm{P}=<0.0001$ and $\mathrm{P}=0.0001$ respectively) while swab cultures from the plain gel yielded a total of 19 microbial isolates from 5 microorganisms.

Conclusion

Low-cost fortification of ultrasound coupling gel with $0.5 \%$ chlorhexidine and $70 \%$ ethyl alcohol renders it hostile to microorganisms encountered at sonology thus preventing nosocomial transmission.
\end{abstract}

Keywords: sonologic examination, ultrasound gel, antimicrobial fortification, nosocomial infections

\section{Introduction}

Hospital infections pose a risk not just to patients but also to health workers. Even in more advanced settings, these phenomena have had profound financial and health implications. ${ }^{1,2}$ There is evidence that the prevalence of certain hospital-acquired pathogens is on the rise worldwide ${ }^{3}$ and some authors have previously implicated medical instruments in germ transmission. ${ }^{4}$ Infection control is vital in radiology departments, which are high traffic areas for patients and hospital staff. ${ }^{5}$ Ultrasound probes are devices repeatedly used in the examination of various patient anatomies with hydrophilic gels used as coupling media. Some authors ${ }^{6,7}$ have averred that the ultrasound probe is a potential vector for the transmission of pathogens such as methicillin-resistant Staphylococcus aureus and Pseudomonas, among others. For routine non-invasive scans, it is our practice to simply wipe the gel off the probe head after scanning using dry soft tissue paper. However, some authors have expressed doubt if this cleaning protocol confers adequate disinfection. ${ }^{8}$ More so it has been found that even where equipment cleaning occurs, there are infrequent low levels of cleaning. ${ }^{9}$ With the recent appearance of severe, life-threatening contagion, it becomes emergent to reinforce existing antimicrobial cleaning protocols and evaluate new ones.

The objective of this study is to evaluate the effectiveness of antimicrobial fortification of non-sterile ultrasound gel using chlorhexidine gluconate ${ }^{10}$ and ethyl alcohol ${ }^{11}$ as an ergonomic innovation to prevent gel-mediated nosocomial infection during sonologic imaging, in a low-resource setting. 


\section{Methods}

This was a pilot study, a cross-sectional comparative analysis, carried out in October 2016 in a busy private radiology clinic in Enugu, Nigeria. The facility receives approximately 100 different referrals per day. Aside from other imaging modalities, there are 4 ultrasound machines operated by 4 different sonologists at any time. Ethical approval for the study was sought and obtained from the Radiation Medicine Human Research Ethical Committee of University of Nigeria Teaching Hospital, Enugu (Radiation Medicine UNTH HREC). Verbal consent was obtained after reading out the consent form in English and if need be, in the patients' local language. The study was carried out on alternate week days over a 2 -week period. A total of 20 consenting adult patients (10 controls and 10 test cases), who had presented to the clinic for abdominal ultrasound examinations for various indications, were recruited into the study. On each scan day, a total of 4 consecutive consenting patients were scanned. Each day, randomization was done by recruiting the first 2 consenting adult patients on queue to each of the ultrasound machines dedicated to either standard plain, non-sterile ultrasonic coupling gel (PG) or fortified gel (FG). Subjects below the age of 18 were excluded from this study. Also excluded were those not referred for ultrasound per abdominis. Adult men and women for abdominal, obstetric or pelvic indications for ultrasound per abdominis were included in the study.

Each day, plain gel (PG) (Begood Sonic Gel, JJ Industry Co. Ltd) was poured into a plastic dispenser from a reservoir. Fresh fortified gel (FG) was prepared in a glass beaker by mixing $500 \mathrm{ml}$ of standard non-sterile ultrasonic coupling gel (Begood ${ }^{\circledR}$ Sonic Gel, JJ Industry Co. Ltd), $50 \mathrm{ml}$ of $0.5 \%$ chlorhexidine and $25 \mathrm{ml}$ of $70 \%$ ethyl alcohol in a ratio of 20:2:1 respectively. The mixture was whisked thoroughly with a glass rod to dissolve the lumps. The resulting mixture had an opaque pale green colour and less sticky texture compared to the PG (Figure 1) and was scooped into a plastic dispenser.

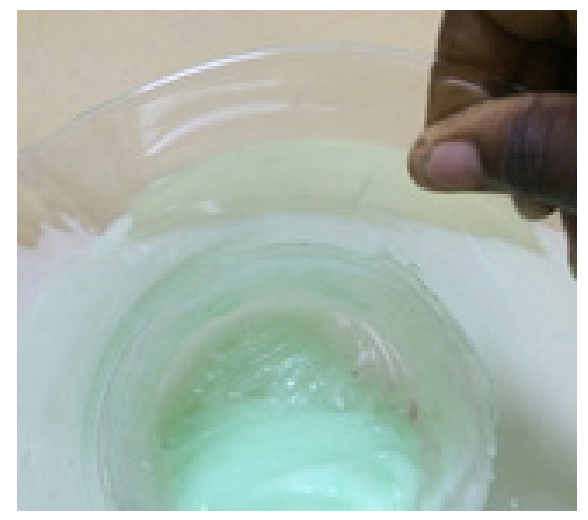

Figure 1: Beaker with the fortified gel made from plain gel, $0.5 \%$ chlorhexidine and $70 \%$ ethyl alcohol in a 20:2:1 composition (note the opaque pale green colour)

The study was divided into two stages, A and B.

Stage $A$ : This stage involved scanning the patients with the gels, PG and FG. We applied about $5 \mathrm{ml}$ of plain coupling gel (PG) over each quadrant of each patient's abdomen and scanned the entire abdomen using the ultrasound transducer. After that, with one sterile swab stick per patient, we took samples (PGA1 series) from the quadrants of each subject's abdomen, as well as from the scan surface of the gel-laden transducer. Then, with the usual commercially-available dry soft tissue paper, we wiped the probe head dry, took a swab of the wiped transducer and labelled it PGA2 series.

For the next 10 patients, applying fortified gel (FG) over each quadrant of each patient's abdomen, we again scanned the entire abdomen. At the end of each scan with fortified gel, we again collected swab samples (FGA1 series) from the four quadrants of the patient's abdomen as well as the surface of the gel-laden transducer. The transducer was again wiped dry with soft tissue paper, and we took another swab of the wiped transducer surface and labelled it FGA2 series. In total, at the end of the study, 10 patients had been scanned with FG (test cases) and 10 with PG (controls). We cultured 10 swab samples each, of the PGA1, PGA2, FGA1 and FGA2 series for 24 hours using Blood and MacConkey agars. Each scan day, the samples were immediately taken to the microbiology laboratory housed within the same facility. Following standard procedures, ${ }^{12}$ a sterile flamed wire loop was used to obtain samples from the swab sticks and inoculated into the agar plates and incubated for 24 hours at $35-37^{\circ} \mathrm{C}$. Inspection and identification of growth was done by the resident microbiologist who was blinded to the source of the specimens.

Stage B: We left separate samples each of plain gel (PG) and fortified gel (FG) to stand in their plastic dispensers for 72 hours in the ultrasound room. We took swab stick samples of the plain gel (PGB) and the fortified gel (FGB) and cultured them aerobically for 24 hours using Blood and MacConkey agars. At the end of the culture, plates were inspected for growth, and any microbial colonies were identified.

Data were analysed using GraphPad Prism, Version 5.03 (Graphpad Software Inc. USA, 1992-2010). Fisher's Exact Test was used to compare outcomes when plain and fortified ultrasonic gels were used on patients. This test was deployed especially because of the small sample size and some expected cell values being less than 1 . A p-value of $<0.05$ was considered statistically significant.

\section{Results}

The agar plates were inspected after 24 hours. Cultures of swabs taken from the 10 patients scanned with the plain standard coupling gel (PGA1 series) yielded a total of 19 microbial isolates from 5 organisms - Staphylococcus epidermidis, Staphylococcus aureus, Psendomonas, Corynebacter spp and Acinetobacter spp (Figure 2). Of these, S. epidermidis was the most common microbe -8 isolates out of $19(42 \%)$. Swabs of the wiped transducer head taken after scanning 10 patients (PGA2 series) yielded 14 microbial isolates similar to those of the PGA1 series except that 1 swab returned with no growth (Figure 2). S. epidermidis was also the commonest isolate occurring in 5 out of $14(35.7 \%)$. All swabs taken from the 10 patients scanned with the fortified ultrasound coupling gel (FGA1 series) yielded no growth of microorganisms (Figure 3). 


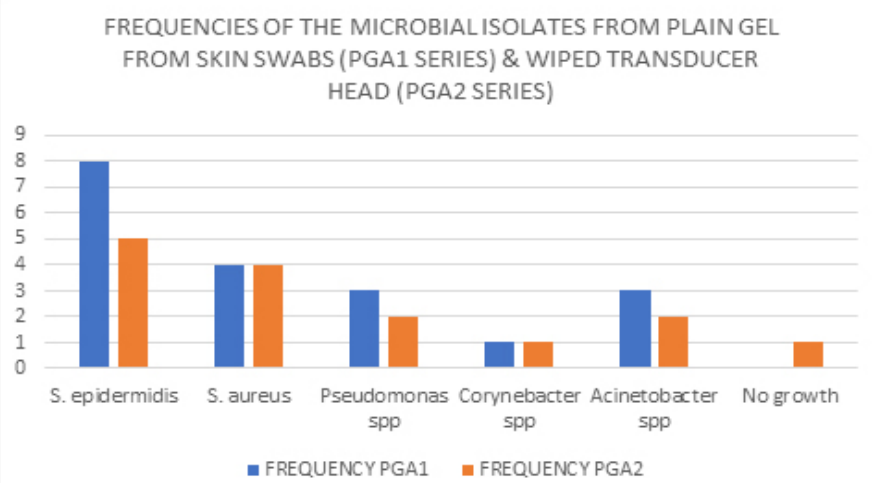

Figure 2: Frequencies of the five microbial isolates from plain gel taken from the patients' abdomen (a total of 19 in PGA1) and that swabbed from the wiped transducer head (a total of 14 in PGA2)

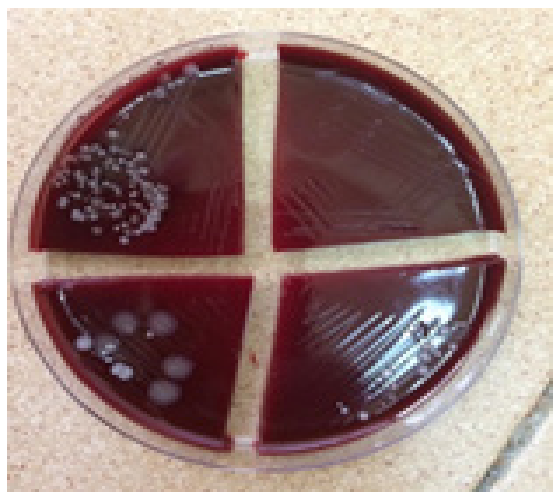

Figure 3: Blood agar plate showing microbial colonies (from the PGA1 series) on all except the right upper quadrant (from the FGA1 series) after 24 hours of aerobic incubation

The same applied to swabs of the wiped transducer head (FGA2 series) (Figure 4).

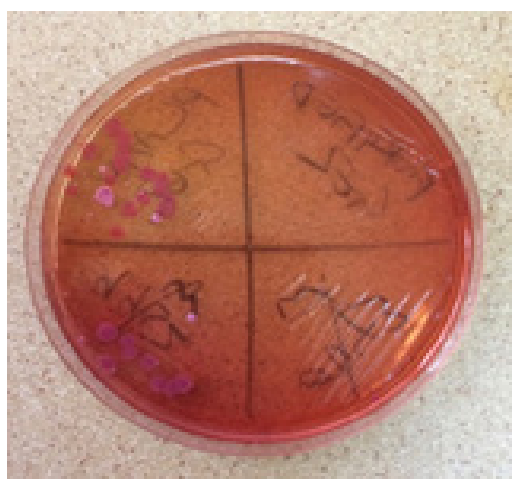

Figure 4: Mac Conkey agar plate showing post-incubation microbial colonies on the two quadrants on the left (from the PGA2 series) and no growth on the two quadrants on the right (FGA2 series)

There was a growth of $S$. aureus from the plain gel sample (PGB) left standing for 72 hours (Figure 5).

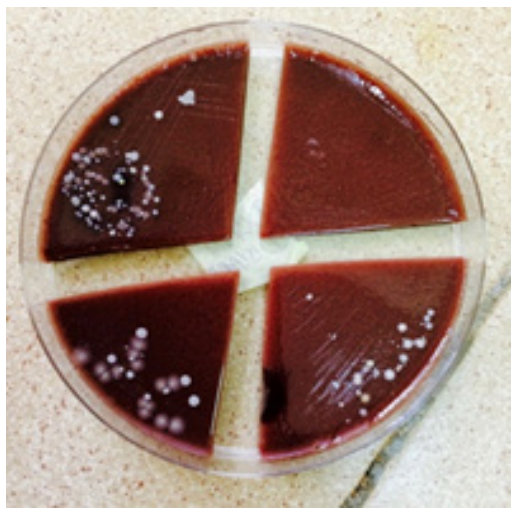

Figure 5: Blood agar plate depicting stage $B$ of the experiment: Showing microbial colonies on three quadrants of the agar plate (from the plain gel dispenser- PGB) except the right upper quadrant (from the fortified gel dispenser- FGB) after 24 hours of aerobic incubation

Meanwhile, no microbial growths were found in the sample taken from the fortified gel (FGB) (Figure $5 \&$ Table 1).

Table 1: Depicting stage B of the study: A comparative assay of microbial growth between plain (PGB) and fortified gel (FGB) left to stand for 72 hours and swab stick samples taken and incubated for 24 hours in nutrient agar; while the plain gel sample was contaminated by $S$. aureus, no growth was recorded with samples of fortified gel.

\begin{tabular}{|l|l|l|l|}
\hline \multicolumn{2}{|l|}{ 72- hour plain gel sample (PGB) } & \multicolumn{2}{l|}{ 72- hour fortified gel sample (FGB) } \\
\hline Nutrient agar used & Growth Yes/No & Nutrient agar used & Growth Yes/No \\
\hline A & Yes (S. aureus) & A & No \\
\hline B & Yes (S. aureus) & B & No \\
\hline
\end{tabular}

Swab stick cultures taken from the gel on the patients' skin and transducer head yielded isolates in all 10 specimens from the plain gel and no observable isolates in all 10 specimens from the fortified gel. Comparison on the contingency table yielded a two-sided P-value of $<0.0001$ indicating that the observed difference in microbial isolates between PGA1 vs FGA1 series are real, thereby testifying to the significant antimicrobial effect of fortified gel (Table 2).

Table 2: Comparison of culture outcomes from swab sticks of plain (PGA1) and fortified (FGA1) ultrasonic gel taken from the skin of the patients; there was microbial growth in all 10 samples taken from the plain gel (PGA1) while no growth was noted in all 10 samples from the fortified gel (FGA1). At $P<$ 0.0001; this difference was statistically significant.

\begin{tabular}{|l|l|l|l|}
\hline & PGA1 & FGA1 & TOTAL \\
\hline GROWTH & 10 & 0 & 10 \\
\hline NO GROWTH & 0 & 10 & 10 \\
\hline TOTAL & 10 & 10 & 20 \\
\hline
\end{tabular}

For the swab stick cultures from wiped transducer heads, there were microbial isolates in 9 out of 10 specimens in the transducers that had plain gel wiped off them and no isolates from the transducer that was wiped off fortified gel (PGA2 vs FGA2 in Table 3). 
Table 3: Comparison of culture outcomes from swabs taken from the wiped transducer heads after using plain (PGA2) and fortified (FGA2) ultrasonic gel on patients. There was microbial growth in 9 out of 10 samples taken from the wiped transducer head used with plain gel (PGA2), while no growth was noted in all 10 samples from the wiped transducer head used with fortified gel (FGA2). At $\mathbf{P}=\mathbf{0 . 0 0 0 1}$; this difference was statistically significant.

\begin{tabular}{|l|l|l|l|}
\hline & PGA2 & FGA2 & TOTAL \\
\hline GROWTH & 9 & 0 & 9 \\
\hline NO GROWTH & 1 & 10 & 11 \\
\hline TOTAL & 10 & 10 & 20 \\
\hline
\end{tabular}

This yielded a two-sided P-value of 0.0001, equally indicating the significant antimicrobial effect of gel fortification on transducer heads. Most importantly, concerning image resolution, there was no apparent difference in quality between the images produced via the different gels, plain and fortified gel (figure 6).

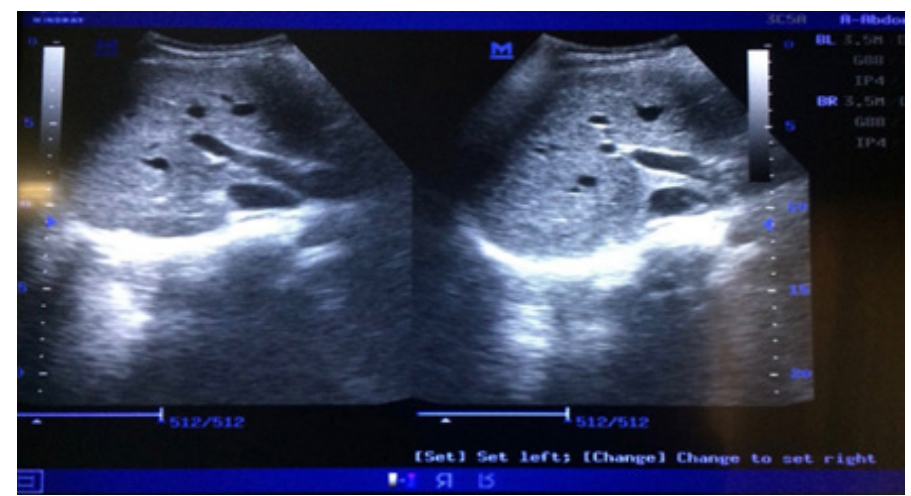

Figure 6: Transverse ultrasound scans of the adult liver showing the comparable image resolution between the image produced through the media of plain gel (left) and that of fortified gel (right) respectively

\section{Discussion}

The fact that the ultrasound coupling gel can be colonised by microbes and possesses no bacteriostatic or bacteriocidal properties has been established. ${ }^{13,14}$ In the constitution of the fortified gel, we added two agents with proven germicidal properties to the standard ultrasound coupling gel, namely chlorhexidine gluconate ${ }^{10}$ and ethyl alcohol. ${ }^{11}$ Chlorhexidine has been used alone or in combination with other agents in sanitising the pre-operative skin. ${ }^{15} \mathrm{It}$ has also been found of use in surgical hand preparation, ${ }^{16}$ in other more invasive procedures like peripheral venous cannulation, ${ }^{17}$ as well as in the sanitization of medical instruments including ultrasound probes. ${ }^{8}$ Ethyl alcohol is lethal to many organisms including viruses. ${ }^{18}$ Alone, it has been effectively used for the sanitisation of transducer heads $^{19}$ and other instruments at $70 \%$ concentration; ${ }^{20}$ however, a combination of chlorhexidine and ethyl alcohol results in an augmentation of antimicrobial activity. ${ }^{21}$ Other authors confirm that the combination of ethyl alcohol and chlorhexidine possesses even greater residual antimicrobial activity compared with ethyl alcohol alone. ${ }^{22}$ Different dilutions of chlorhexidine with ethyl alcohol have been used successfully by other workers to harness the power of their antimicrobial synergy. ${ }^{15}$ In our experiment, we diluted 1 part $(25 \mathrm{ml})$ of $70 \%$ ethyl alcohol with two parts $(50 \mathrm{ml})$ of $0.5 \%$ chlorhexidine, adding this mixture to 20 parts $(500 \mathrm{ml})$ of standard hydrophilic gel. Having tried other dilutions, we settled for a 20:2:1 gel-chlorhexidine-ethyl alcohol ratio in the bid to confer antimicrobial property and inhibit the growth of microbes without adversely affecting gel consistency. We demonstrated, by the fact that there was no microbial growth in post-scan samples, that, with fortified gel, we required no other special cleaning protocol besides using dry soft tissue paper. Comparatively, the swab samples from the plain gel series (PGA1 and PGA2) yielded growth of microorganisms (See figure 2). Our study concurs with Ohara et al. ${ }^{6}$ who demonstrated that merely cleaning the probes with a dry towel was insufficient sanitisation. However, a similar study by Muradali et al. ${ }^{8}$ concluded that dry towel cleaning conferred sufficient sanitisation. Even if this was true, it, however, remains incontrovertible that standard gel inadvertently left behind on the probe forms a potential nidus of infection for the sonologist and subsequent patients. With fortified gel, however, the potential of left-over gel being a potential breeding site for microbes is obviated. As observed in our study, plain gel not only served as a conducive environment for the growth of skin flora, but also independently permitted growth of germs on standing. This observation has been confirmed by other authors. ${ }^{8}$ A study once reported that an outbreak of infection in a hospital was eventually traced to the ultrasound coupling gel. ${ }^{23}$ Therefore, plain gel is potentially susceptible to colonisation by pathogens. ${ }^{19} \mathrm{It}$ can also serve as a vector for the spread of multiple resistant skin pathogens. ${ }^{24}$ However, in our study, the growth of microbes was effectively inhibited in fortified gel. Although our work did not test the virucidal utility of the fortified gel, the potential for this can be broadly extrapolated in the light of the proven virucidal properties of ethyl alcohol ${ }^{18}$ and chlorhexidine. ${ }^{10}$ One could be worried about the potential for physical degradation of the transducer head by the fortified gel. However, this concern has been put to rest by the work of Shukla et al. who reported no damaging effect to the transducer when repeatedly wiped with $2 \%$ chlorhexidine $/ 70 \%$ alcohol wipes. ${ }^{25}$

\section{Study limitations}

The limitations of this study include the small sample size, this being a pilot study. More importantly, a randomized controlled trial would have been the most suitable study design for this work. Also, there was a potential for selection bias due to our inability to perform pre-procedural skin swabs and non-inclusion of subjects' demographic data- especially age and sex. This being a small study, minimum inhibitory concentration tests were not done. These shortcomings can be resolved in further studies with larger sample size and a wide variation of subjects in order to determine the minimum inhibitory concentration of the additives required to confer bactericidal properties to ultrasound gel. This will help in conservation of resources especially in resourceconstrained environments.

\section{Conclusion}

The fortification of standard ultrasound coupling gel 
with $0.5 \%$ chlorhexidine and $70 \%$ ethyl alcohol renders it hostile to microbes encountered during routine ultrasound examinations. It can, therefore, prevent probe-head transmission of infection-causing bacteria. The use of fortified gel delivers probe sanitisation in non-critical settings without any degrading effect on image resolution.

\section{Conflict of interest}

The authors declare that there are no conflicts of interest regarding the conceptualization and execution of this study.

\section{References}

1. Wenzel RP, Edmond MB. The impact of hospital-acquired bloodstream infections. Emerg Infect Dis. 2001;7(2):174-177. doi:10.3201/eid0702.700174.

2. Cosgrove SE, Qi Y, Kaye KS, Harbarth S, Karchmer AW, Carmeli Y. The Impact of Methicillin Resistance in Staphylococcus aureus Bacteremia on Patient Outcomes: Mortality, Length of Stay, and Hospital Charges. Infection Control \& Hospital Epidemiology. Cambridge University Press; 2005;26(2):166-74. https://doi.org/10.1086/502522. 3. Boyce JM, Cookson B, Christiansen K, Hori S, Vuopio-Varkila J, Kocagoz S, et al. Methicillin-resistant Staphylococcus aureus. Lancet Infect Dis. 2005;5(10):653-663. doi: 10.1016/S1473-3099(05)70243-7. 4. Spach DH, Silverstein FE, Stamm WE. Transmission of infection by gastrointestinal endoscopy and bronchoscopy. Ann Intern Med. 1993;118(2):117-128. doi: 10.7326/0003-4819-118-2-19930115000008 .

5. Zhang E, Burbridge B. Methicillin-resistant Staphylococcus aureus: Implications for the radiology department. Am J Roentgenol. 2011;197(5):1155-9. doi 10.2214/AJR.11.6584.

6. Ohara T, Itoh Y, Itoh K. Ultrasound instruments as possible vectors of staphylococcal infection. J Hosp Infect. 1998;40(1):73-77. https://doi. org/10.1016/S0195-6701(98)90028-7.

7. Mullaney PJ, Munthali P, Vlachou P, Jenkins D, Rathod A, Entwisle J. How clean is your probe? Microbiological assessment of ultrasound transducers in routine clinical use, and cost-effective ways to reduce contamination. Clin Radiol. 2007;62(7):694-698. https://doi. org/10.1016/j.crad.2007.01.002.

8. Muradali D, Gold WL, Philips A, Wilson S. Can ultrasound probes and coupling gel be a source of nosocomial infection in patients undergoing sonography? An in vivo and in vitro study. Am J Roentgenol. 1995;164(6):1521-1524. doi: 10.2214/ajr.164.6.7754907.

9. Núñez S, Moreno A, Green K, Villar J. The stethoscope in the emergency department: A vector of infection? Epidemiol \& Infect. 2000;124(2):233-237.

10. Hosobuchi K, Sato K, Kobayashi H, Takayashi Y, Tsuzuki M. Adsorption of chlorhexidine to hands during hand wash and antibacterial activity of adsorbed chlorhexidine. J Antibaci Antifung Agents. 1981;9:515-519.

11. Spaulding EH. Alcohol as a surgical disinfectant: Pros and cons of a much discussed topic. AORN Journal. 1964;2 (5):67-71. https://doi. org/10.1016/S0001-2092(08)70453-8.

12. Barrow GI, Feltham RKA, editors. Quality control in microbiology. In: Cowan and Steel's Manual for the Identification of Medical Bacteria. 3rd ed. Cambridge: Cambridge University Press; 1993. p. 184-7.

13. Mattar EH, Hammad LF, Ahmad S, El-kersh TA. An investigation of the bacterial contamination of ultrasound equipment at a University
Hospital in Saudi Arabia. J Clin Diagn Res. 2010;4(4):2685-2690.

14. Akpochafor MO, Eze CU, Adeneye S, Ajekigbe . Assessment of ultrasound equipment as a possible source of nosocomial infection in Lagos state hospitals and radio-diagnostic centres. Radiography 2010; 21(2):154 -159. https://doi.org/10.1016/j.radi.2014.09.008.

15. Darouiche RO, Wall MJ Jr, Itani KM, Otterson MF, Webb AL, Carrick MM, et al. Chlorhexidine-alcohol versus povidone-iodine for surgical-site antisepsis. N Engl J Med. 2010;362(1):18-36. doi: 10.1056/NEJMoa0810988.

16. Le TA, Dibley MJ, Vo VN, Archibald L, Jarvis WR, Sohn AH. Reduction in surgical site infections in neurosurgical patients associated with a beside hand hygiene program in Vietmnam. Infect Control Hosp Epidemiol. 2007;28(5):583-588. doi: 10.1086/516661.

17. Valles J, Fernandez I, Alcaraz D, Chacon E, Cazorla A, Canals $\mathrm{M}$, et al. Prospective randomized trial of 3 antiseptic solutions for prevention of catheter colonisation in an intensive care unit for adult patients. Infect Control Hosp Epidemiol. 2008;29(9):847-853. https:// doi.org/10.1086/590259.

18. Ali Y, Dolan MJ, Fendler EJ, Larson EL. Alcohols. In: Block SS, editor. Disinfection, sterilization, and preservation. Philadelphia: Lippincott Williams \& Wilkins;2001. p.229-254.

19. Schabrun S, Chipchase L, Rickard H. Are therapeutic ultrasound units a potential vector for nosocomial infection? Physiother. Res. Int. 2006;11(2):61-71. https://doi.org/10.1002/pri.329.

20. Lambert I, Tebbs SE, Hill D, Moss HA, Davies AJ, Elliott TSJ. Interferential therapy machines as possible vehicles for cross-infection. $\mathrm{J}$ Hosp Infect. 2000;44(1):59-64. https://doi.org/10.1053/jhin.1999.0647.

21. Sogawa Y, Kobayashi H, Kajiura T, Nishihara Y. Comparison of residual antimicrobial activity of chlorhexidine-containing antiseptics: An express report. J Healthcare-Assoc Infect. 2010;2:32-36.

22. Reichel M, Heisig P, Kohlmann T, Kampf G. Alcohols for skin antisepsis at clinically relevant skin sites. Antimicrob Agents Chemother. 2009;53(11):4778-4782. doi: 10.1128/AAC.00582-09.

23. Gaillot O, Maruéjouls C, Abachin É, Lecuru F, Arlet G, Simonet M, et al. Nosocomial outbreak of Klebsiella pneumoniae producing SHV5 extended-spectrum beta-lactamase, originating from a contaminated ultrasonography coupling gel. J Clin Microbiol. 1998;36(5):1357-1360.

24. Bello TO, Taiwo SS, Oparinde DP, Hassan WO, Amure JO. Risk of nosocomial bacteria transmission: evaluation of cleaning methods of probes used for routine ultrasonography. West Afr J Med. 2005;24(2):167-170. http://dx.doi.org/10.4314/wajm. v24i2.28190.

25. Shukla B, Howell V, Griffiths A, Thoppil A, Liu M, Carter J, et al. Superiority of chlorhexidine $2 \% /$ alcohol $70 \%$ wipes in decontaminating ultrasound equipment. Ultrasound. 2014; 22(3): 135-140. https://doi. org/10.1177/1742271X14529761. 